\title{
Handedness and Behavioural Inhibition: Left-handed females show most inhibition as measured by BIS/BAS self-report
}

\author{
Lynn Wright ${ }^{1}$, Scott M. Hardie, Karen Wilson \\ Centre for Psychology, University of Abertay Dundee, Bell Street, Dundee, DD1 \\ 1HG, UK
}

\begin{abstract}
This study investigated the relationship between handedness, gender and behavioural approach and inhibition using Carver and White's (1994) BIS/BAS Scale. 112 participants took part: 46 left-handers and 66 right-handers. All participants completed Peters' (1998) handedness questionnaire followed by the self-report BIS/BAS Scale. Significant effects of both handedness and gender on the BIS scores were found, with left-handers and females scoring significantly higher on inhibition. BIS scores were re-examined to include FFFS scores, which showed a significant effect of gender. Revised BIS scores replicated the original BIS findings. These findings are discussed in relation to handedness research.
\end{abstract}

Keywords: Handedness, Gender, Behavioural Inhibition (BIS), Behavioural Activation (BAS), Anxiety, RST.

First copyedit complete.

Introduction

Converging evidence suggests the right-hemisphere of the brain is more involved in processing negative emotional information and right-hemisphere activation is associated with temperamental shyness, anxiety, and behavioural inhibition in human infants (Schmidt, Fox, Schulkin \& Gold, 1999), and behavioural inhibition in primates' motor responses to novel objects (E.g. Cameron \& Rogers, 1999). Thus, research consistently indicates that right hemisphere dominance, or activation, can result in behavioural inhibition. This has been supported by work relating physiology to emotional asymmetry (e.g. Davidson, 1985, 1998). Gray (1982) originally suggested that we have two independent neural behavioural systems - the Behavioural Inhibition System (BIS) and the Behavioural Activation System (BAS). These two

\footnotetext{
${ }^{1}$ Corresponding author, Tel. +44 1382 308587, fax +44 1382308749

E-mail address: 1.wright@abertay.ac.uk (L.Wright)
} 
systems are motivational systems that influence our underlying behaviour, relating to either anxiety (BIS) or impulsivity (BAS). The BIS system relates to avoidance behaviour while the BAS relates to approach behaviour (Carver \& White, 1994). Individuals can experience a number of combinations of BIS/BAS sensitivity (e.g. high BAS sensitivity but low BIS sensitivity or low BIS and low BAS sensitivity). Therefore if one specific stimulus was shown to a group of people they would react in a number of different ways depending on which of the behavioural systems were activated and to what extent (Gray, 1981). Carver and White (1994) state that when the BIS is activated the individual may become behaviourally inhibited. One reason cited for the activation of the BIS system is to avoid any form of confrontation or negative outcome but by doing so the individual may miss the chance to gain positive outcomes or rewards. Gray (1981) states that BIS activation makes the individual experience negative feelings such as frustration, fear and anxiety and this, in turn, will influence behaviour. Stuettgen, Hennig, Reuter and Netter (2005) report that individuals with high BIS scores may be prone to high levels of anxiety, adding that these individuals possibly adopt a series of behavioural coping strategies in order to reduce their anxiety levels.

Behavioural response style differences have been linked to hemispheric differences. Davidson (1985; 1998) proposed that the BIS system was a withdrawal system, predominantly linked to the right hemisphere relating to inhibition of behaviour and negative affect. He argues that the left hemisphere is an activation system, linked to positive affect and approach. Other evidence suggests that BIS is more related to processing of threat, rather than anxiety per se (e.g. Avila \& Torrubia, 2006), and is arguably a threat detection system. This suggests that BIS is not purely a measure of anxiety but a system related to behavioural inhibition that may be influenced by anxiety levels. Linking this to the BIS/BAS questionnaire, several studies have shown that cortical activity in the left frontal lobe has been linked to increased BAS scores (Coan \& Allen, 2003; Sutton \& Davidson, 1997). Taken together, this suggests that Carver and White's (1994) BIS/BAS questionnaire is tapping into a measure of response style (i.e. approach/avoidance) that is intrinsically linked to hemispheric activation. This allows the prediction of dispositional differences related to handedness differences.

To our knowledge, very little previous research exists linking handedness and Behavioural Inhibition/Activation. However, several have investigated the relationship between anxiety and handedness. Hicks and Pellegrini (1978), 
Davidson and Schaffer (1983) and Wright (2005) reported that left-handers were more anxious than right-handers, whereas studies such as Beaton and Moseley (1984) and Merckelbach, de Ruiter and Olff (1989) have found no relationship between anxiety and handedness. It is therefore interesting to investigate any possible relationship between handedness and behavioural inhibition given the strong link between behavioural inhibition and anxiety. Previous studies have investigated the relationship between handedness and behavioural response through approach and avoidance behaviour (e.g. Wright, Hardie \& Rodway, 2004; Cameron \& Rogers, 1999). A strong link between left-handedness and avoidance behaviour was reported, where left-handed participants took significantly longer to approach an object or task than right-handed individuals. One explanation given in the study by Cameron and Rogers (1999) is that the novelty of a situation might have influenced left-handers more than right-handers. Wright et al. (2004) also reported that the task novelty may have been related to the avoidance behaviour shown by lefthanded participants in their study. This effect of novelty and avoidance can be related to behavioural inhibition. Quilty, Oakman and Farvolden (2007) state that behavioural inhibition is positively related to a preference for familiarity and behavioural activation is negatively associated with familiarity, with novelty seeking being one central feature of the BAS system. Thus this association would cause us to speculate that there might be a link between handedness and approach and avoidance behaviour as measured by the BIS/BAS scale.

Recent work (e.g. Smillie, Pickering \& Jackson, 2006) has argued that Carver and White's scale does not reflect the extensive theoretical changes since Gray's original theory. Specifically, Gray \& McNaughton (2000) extended the original idea of impulsivity (BAS) and anxiety (BIS) into a three system theory. This revised theory (now generally referred to as Reinforcement Sensitivity Theory, RST) both modified the original BIS concept, and added a Fight-Flight-Freeze System (FFFS), which was thought to relate to how to respond to aversive stimuli, mainly via avoidance, either defensive (fear) or escape (panic). The BAS system is still defined as an approach system, but the BIS system is now not an avoidance system per se, but is re-defined as a system related to resolving goal conflict (e.g. approach vs. avoidance) which includes conflict both within and between the systems. Thus BIS can be considered to be inhibiting ongoing behaviour (FFFS and BAS mediated behaviour) and the same time directing attention and arousal towards the stimuli causing the conflict (Smillie et al., 2006). This generates a state of anxiety and leads to the assessment of risk and resolution of the conflict (see Corr \& McNaughton, 2008). 
Therefore, the clearest implication of this work is that there should be a distinction between fear (via FFFS) and anxiety (via BIS), and applying this to handedness might help to explain behavioural differences. For example, when left-handers take longer to start a task (e.g. Wright et al., 2004), we will be able to hypothesise about the relative role of fear and anxiety in terms of what may be creating the delay to action. A higher BIS score would support the idea of left-handers being more anxious about wanting to approach it, rather than showing fear from the stimuli.

To date, however, few studies have tested the revised RST (mainly due to Carver \& White's (1994) BIS/BAS scales not mapping directly onto it), but recent work (Corr \& McNaughton, 2008) has shown that FFFS scores can be derived from BIS questions. This will be explored in the current study.

A link can also be made between gender and behavioural inhibition. Numerous studies report a relationship between gender and anxiety, and it is clear that females are more anxious than males (e.g. Feingold, 1994; MacKinaw-Koons \& Vasey, 2000). Several studies have investigated the possible relationship between gender and behavioural inhibition, and have consistently shown that females score higher on BIS scores (Carver \& White, 1994; Leone, Perugini, Bagozzi, Pierro \& Mannetti, 2001). In addition, while Mardaga and Hansenne (2007) also reported that females scored significantly higher on the BIS scale they found that their BAS scores were very similar to males (overall and for all 3 sub-scales). Similarly, Jorm et al. (1999) reported the same pattern in BIS scores but found that females scored higher on the BAS reward responsiveness scale while males scored significantly higher on the BAS drive scale. This suggests that there is usually a clear gender difference in BIS scores, but no consistent pattern in BAS scores.

The present study examines the relationship between handedness, gender and selfreport BIS/BAS (including a derived measure of FFFS to reflect revisions to RST) using Peters' (1998) handedness questionnaire and Carver and White's (1994) BIS/BAS scale. As left-handers exhibit right hemisphere dominance, linked to the brains' inhibitory system, it is hypothesised that the BIS scores of left-handers will be higher than those of right-handers. As right-handers exhibit left hemisphere dominance linked to the behavioural activation system, it is hypothesised that righthanders will score higher on BAS. Additionally, it is hypothesised that females will have higher BIS scores. 


\section{Method}

\section{Participants}

112 participants took part in this study - 54 males and 58 females. 46 participants were left-handed ( 24 males and 22 females) and 66 participants were right-handed (33 males and 33 females). All were University students with an age range from 18 to 52 years.

\section{Materials}

Peters' (1998) handedness questionnaire was used to measure participant's handedness. This is a 25 item scale scored using a 5 point Likert scale (left-hand always, left-hand mostly, either hand, right-hand mostly and right-hand always). The five points on the scale are assigned values from -2 (always use the left hand) through to 2 (always use the right hand) and each item is scored individually then totalled to give an overall handedness score. A total positive value indicates a righthand preference and a total negative value indicates a left-hand preference. The BIS/BAS questionnaire (Carver \& White, 1994) consisted of a list of 24 statements which participants were asked whether they agreed or disagreed with (using a 4point Likert scale). Examples of statements are 'criticism or scolding hurts me quite a bit' and 'when good things happen to me, it affects me strongly'. Participants were instructed to respond to all items and not leave any blank. The scoring system measured the participants' Behavioural Activation levels (3 sub-sections) and Behavioural Inhibition levels.

\section{Procedure}

Participants were asked to complete the handedness questionnaire followed by the BIS/BAS questionnaire. Participants were asked to read the instructions carefully and were informed that there was no time limit. Total BAS, BAS Drive; BAS Fun seeking; BAS Reward and BIS scores were calculated along with handedness questionnaire scores. Additionally, the BIS scale was broken down, and scored, into FFFS (questions 2 and 22) and BIS components (remaining 5 original BIS questions) (See Corr \& McNaughton, 2008). For handedness participants were divided into the broad groups of 'left-handed' and 'right-handed' where a right-hander was classed as someone scoring a total positive score and a left-hander was classed as someone scoring a total negative score. 


\section{Results}

\section{Handedness Questionnaire}

\section{(Table 1 here)}

The above table shows that both left- and right-handers showed a strong hand preference, where a stronger hand preference is indicated by a higher absolute value for the handedness score.

Independent t-tests showed no significant differences between male and females' handedness scores (both overall and within handedness categories), $\underline{p}>0.05$ for all comparisons.

\section{BIS/BAS Questionnaire}

7 measurements were taken from the questionnaire and are reported sequentially below.

\section{BAS Results}

\section{(Table 2 here)}

Table 2 indicates that total BAS scores were similar across all participant groups.

A $2 \times 2$ (handedness (left vs. right) by gender (male vs. female)) ANOVA was carried out on total BAS scores. There was no significant main effect of handedness $\underline{F}(1$, $108)=1.49, \underline{p}>0.05$. There was no significant main effect of gender $\underline{F}<1$ and the interaction between sex and handedness failed to reach significance $\underline{F}(1,108)=$ $1.43, \underline{p}>0.05$.

\section{BAS Drive}

A $2 \times 2$ (handedness (left vs. right) by gender (male vs. female)) ANOVA was carried out on BAS Drive scores. There was no significant main effect of handedness $\underline{E}(1$, $108)=2.15, \underline{p}>0.05$ There was no significant main effect of gender $\underline{F}<1$, and the 
interaction between sex and handedness failed to reach significance $\underline{F}(1,108)=$ $2.57, \underline{p}=>0.05$

\section{BAS Fun seeking}

A $2 \times 2$ (handedness (left vs. right) by gender (male vs. female)) ANOVA was carried out on BAS Fun seeking scores. There was no significant main effect of handedness $\underline{F}(1,108)=1.65, \underline{p}>0.05$. There was, however, a significant main effect of gender $\underline{F}(1,108)=4.70, \underline{p}<0.05$ (partial $\eta^{2}=0.04$ and an observed power of 0.58 ) with males scoring significantly higher than females. The interaction between sex and handedness was not significant $\underline{F}(1,108)=1.02$, $\underline{\mathrm{p}}>0.05$.

\section{BAS Reward responsiveness}

A $2 \times 2$ (handedness (left vs. right) by gender (male vs. female)) ANOVA was carried out on BAS Reward responsiveness scores. There was no significant main effect of handedness $\underline{F}<1$. There was no significant main effect of gender $\underline{F}<1$ and the interaction between sex and handedness also failed to reach significance $\underline{F}<1$.

\section{BIS Results}

\section{(Table 3 here)}

Female left-handers scored the highest average BIS score (they scored on average 24 out of a possible 28).

A $2 \times 2$ (handedness (left vs. right) by gender (male vs. female)) ANOVA was carried out on BIS scores. There was a significant main effect of handedness $\underline{F}(1,108)=$ 8.94, $\underline{\mathrm{p}}<0.01$ (partial $\eta^{2}=0.08$ and an observed power of 0.89 ) with left-handers scoring significantly higher than right-handers. There was also a significant main effect of gender $\underline{F}(1,108)=15.18, \underline{p}<0.01$, (partial $\eta^{2}=0.12$ and an observed power of 0.97 ) with females scoring significantly higher than males. However, the interaction between sex and handedness failed to reach significance $\underline{F}<1$. 


\section{Revised BIS and FFFS results}

\section{(Table 4 here)}

When the BIS scale was split in to revised BIS and FFFS components female lefthanders scored the highest, on average, on both scales.

The $2 \times 2$ ANOVA carried out on the revised BIS scores replicated the findings outlined in the original BIS analysis.

A $2 \times 2$ (handedness (left vs. right) by gender (male vs. female)) ANOVA was carried out on FFFS scores. There was a significant main effect of gender $\underline{F}(1,108)=$ 9.58, $\underline{\mathrm{p}}<0.01$ (partial $\eta^{2}=0.08$ and an observed power of 0.86 ) with females scoring significantly higher on FFFS than males. There was no significant main effect of handedness $\underline{F}(1,108)=2.69$, $\underline{p}>0.05$ and the interaction between handedness and gender failed to reach significance $\underline{F}<1$.

\section{Discussion}

This study investigated behavioural activation and inhibition using Carver and White's (1994) BIS/BAS Scale in relation to handedness and gender. It was hypothesised that right-handers would score higher on the BAS questions but this was not supported. For BIS scores there was a significant effect of handedness with lefthanders scoring higher (in both original and revised BIS scores). This supports previous research as the Behavioural Inhibition System has been related to inhibited and anxious behaviour (Gray \& McNaughton, 2000) and left-handers show more anxiety (e.g. Davidson \& Schaffer, 1983; Wright, 2005). Our previous work (Wright et al., 2004) has shown that left-handers were inhibited in their behavioural response to a novel task, suggesting that actual behaviour and response style may be linked to handedness. This work fits well with the suggestions of Carver and White (1994), who reported that the BIS reacts to novel objects and situations and the presence of such stimuli causes the individual to become inhibited in their behaviour in some way. Adding this to the revised RST notion of the role of the BIS suggests that compared to right-handers, left-handers may experience a greater degree of conflict, anxiety and risk assessment when interacting with situations (Gray \& McNaughton, 2000). Thus, we have converging evidence to suggest that in both self-report (this 
study) and actual behaviour (e.g. Wright et al., 2004) that the response style of lefthanders is more likely to be behaviourally inhibited. This adds to the growing body of evidence reporting left-handers to be a generally more anxious or inhibited population, than right-handers. This was also supported in a series of experiments involving state anxiety scores (Wright, 2005) where left-handers reported themselves in the test/experimental situation to feel more anxious than right-handers (particularly when the task was novel). However, this effect was not found for trait anxiety, suggesting that state anxiety may be a sign reflecting BIS's role in risk assessment and the resolution of conflict (Corr \& McNaughton, 2008).

This handedness related behavioural difference may be particularly strong when dealing with novel situations, where left-handers would presumably be more anxious due to their right hemisphere dominance (Schmidt et al., 1999; Cameron \& Rogers, 1999). If the aspect of novelty is combined with the prospect of a task to complete in which the participant does not know the outcome then high levels of anxiety would be displayed by someone with high BIS sensitivity (e.g. Stuettgen et al., 2005). As mentioned above, conceptually this relates to Gray and McNaughton's (2000) revised BIS, in which they argue that it is a system for risk assessment and caution and so lack of approach is presumably related to conflict between wanting to do the task and fear about the outcome (i.e. BAS vs. FFFS). This also relates well to the notion that the revised BIS is related to cautious approach and not fearful avoidance (Corr \& McNaughton, 2008). Therefore, the BIS sensitivity of left-handers may be the underlying reason for reports of anxiety differences between left- and right-handers (e.g. Hicks \& Pellegrini, 1978; Davidson \& Schaffer, 1983). Additionally, when the FFFS components of the BIS scale were analysed in the current study the effect of handedness was not significant supporting the separability of fear (FFFS) and anxiety (BIS).

This would, however, not necessarily mean that task performance was poorer, as in a review of anxiety and cognitive performance, Eysenck, Derakshan, Santos, and Calvo (2007) argued that for anxious people, compensatory strategies may counter any potential negative effects. In our previous work (Wright et al., 2004) we hypothesised that left-handers use 'coping strategies' such as planning to reduce anxiety in novel and/or difficult situations. This gives a plausible theoretical basis for the role of the revised BIS in explaining handedness differences, where left-handers deal with conflict between the goals of other systems by making use of previous 
strategies from memory and assessing the current situation (Corr \& McNaugton, 2008). The self-reported BIS differences found here would support the contention that left-handers are aware of their own (inhibitory) response style and indeed the BIS scale is an indicator of predisposition to, rather than a measure of, anxiety (Jorm et al., 1999). The present study adds to our increasing understanding of behavioural differences related to handedness (e.g. Wright, 2005), and the complex relationship found between anxiety, inhibition and behaviour.

It was also hypothesised that females would have higher BIS scores than males as they are often reported to have higher anxiety levels (Feingold, 1994; MacKinawKoons \& Vasey, 2000). This was supported in this study as females scored significanlty higher BIS scores (revised and original) than males. This fits in well with the findings of many other studies (Carver \& White, 1994; Gard \& Kring, 2007; Jorm et al., 1999), as well as our own behavioural studies (e.g. Wright et al., 2004). Females also had significantly higher FFFS scores than males supporting recent findings (Perkins, Kemp \& Corr, 2007).

No differences were hypothesised between male and female BAS scores, and no significant effects of handedness were found on any of the sub-scales or the total BAS score. However, males scored higher on the BAS fun seeking scale (although the power of this effect was relatively low). The lack of an overall effect is in line with several other recent studies. Scholton, van Honk, Aleman and Kahn (2006), Mardaga and Hansenne (2007) and Jorm et al. (1999) all found no significant difference between normal males and females in their combined BAS scores. Looking at BAS subscales, Jorm et al. (1999) found significantly higher drive scores in males, and reward responsiveness scores in females which were not found in the current study. This suggests that in our sample, males may be more prone to be risk takers than females, and this association has been made in other studies (e.g. Suhr \& Tsanadis, 2007).

Although Gray (1982) originally related BAS to impulsivity, we did not find higher scores related to the left hemisphere dominant (right-handed) participants and so this appears to be unrelated to handedness. One reason for this might be that impulsivity and behavioural activation are not related in a straightforward way (Quilty \& Oakman, 2004; Gray \& McNaughton, 2000). In any case, the role of BAS in response style differences related to both gender and handedness needs further investigation. 


\section{Limitations}

As previously noted there are potential problems with the BIS/BAS questionnaire and its relationship to the constructs of revised RST (Corr \& McNaughton, 2008), as well as there being concerns about the validity of the instrument (e.g. Cogswell, Alloy, van Dulmen \& Fresco, 2006; Gomez, Cooper \& Gomez, 2005).

Additionally, although use of self-report handedness questionnaires has been criticized, this is the most widely used method of quantifying handedness and the reliability statistics for Peters' (1998) questionnaire are excellent (test-retest $r=0.88$ and Cronbach's alpha for the reliability of items $=0.99$ ) (Peters, 2008). In this case, we also have supporting evidence from behavioural differences related to handedness (Wright et al., 2004) and so it may be argued that this strengthens the validity of the current findings.

We should also consider the extent to which Corr and McNaughton's (2008) separation of Carver and White's BIS scale in to FFFS and BIS components is a valid and appropriate measure of these revised RST components

Finally, claims are being made here about brain dominance and lateralisation. It would be useful to make use of fMRI techniques in order to specifically examine the contribution that each hemisphere makes towards inhibition and activation behaviour, in relation to handedness.

\section{Conclusion}

The results of self-reported BIS scores (original and revised) suggest that this system may play a crucial role in the expression of behavioural differences between left- and right-handers, and males and females. 


\section{References}

Avila, C., \& Torrubia, R. (2006). Personality differences in suppression of behavior as a function of the probability of punishment. Personality and Individual Differences, 41, 249-260.

Beaton, A. A., \& Moseley, L. G. (1984). Anxiety and the measurement of handedness. British Journal of Psychology, 75, 275-278.

Cameron, R., \& Rogers, L. J. (1999). Hand preference of the Common Marmoset (Callithrix jacchus): Problem solving and responses in a novel setting. Journal of Comparative Psychology, 113, 149-157.

Carver, C. S., \& White, T. L. (1994). Behavioral inhibition, behavioral activation and affective responses to impending reward and punishment: the BIS/BAS Scales. Journal of Personality and Social Psychology, 67, 319-333.

Coan, J. A. \& Allen, J. J. B. (2003). The state and trait nature of frontal EEG asymmetry in emotion. In K. Hugdahl, \& R. J. Davidson (Eds), Asymmetrical Brain. (pp. 565-615). Cambridge: MA MIT Press.

Cogswell, A., Alloy, L.B., van Dulmen, M.H.M., \& Fresco, D.M. (2006). A psychometric evaluation of behavioral inhibition and approach self-report measures Personality and Individual Differences, 40, 1649-1658 
Corr, P. J. \& McNaughton, N. (2008). Reinforcement Sensitivity Theory and personality. In P. J. Corr (Ed), The Reinforcement Sensitivity Theory of Personality. Cambridge: Cambridge University Press.

Davidson, R. J. (1985). Affect, cognition, and hemispheric specialization. In C.E. Izard, \& J. Kagan, (Eds), Emotions, Cognition, and Behavior (pp. 320-365). New York: Cambridge University Press.

Davidson, R. J. (1998). Affective style and affective disorders: perspectives from affective neuroscience. Cognition and Emotion, 12, 307-330.

Davidson, R. J. \& Schaffer, C. E. (1983). Affect and disorders of affect. In P. FlorHenry, \& J. Gruzelier (Eds.), Hemisphere asymmetries of function in psychopathology (pp. 248-268). New York: Elsevier.

Eysenck, M. W., Derakshan, N., Santos, R., \& Calvo, M. G. (2007). Anxiety and Cognitive Performance: Attentional Control Theory. Emotion, 7 (2), 336-353.

Feingold, A. (1994). Gender differences in personality: A meta-analysis. Psychological Bulletin, 116 (3), 429-256.

Gard, M. G. \& Kring, A. M. (2007). Sex Differences in the Time Course of Emotion. Emotion, 7 (2), 429-437 
Gomez, R., Cooper, A., \& Gomez, A. (2005). An item response theory analysis of the Carver and White (1994) BIS/BAS Scales. Personality and Individual Differences, 39, 1093-1103.

Gray, J. A. (1982). The neuropsychology of anxiety: An enquiry of the septohippocampal system. Oxford, UK: Oxford University Press.

Gray, J. A. (1981). A critique of Eysenck's theory of personality. In H. J. Eysenck (Ed.), A model for personality (pp. 246-277). Berlin: Springer.

Gray, J. \& McNaughton, N. (2000). The neuropsychology of anxiety (2 ${ }^{\text {nd }}$ ed.). Oxford, UK: Oxford University Press.

Hicks, R. A., \& Pellegrini, R. J. (1978). Handedness and anxiety. Cortex, 14, 119121.

Jorm, A. F., Christensen, H., Henderson, A. S., Jacomb, P. A., Korten, A. E., \& Rodgers, B. (1999). Using the BIS/BAS scales to measure behavioral inhibition and behavioral activation: Factor structure, validity and norms in a large community sample. Personality and Individual Differences, 26, 49-58.

Leone, L., Perugini, M., Bagozzi, R. P., Pierro A. \& Mannetti, L. (2001). Construct validity and generalizability of the Carver-White behavioural inhibition system/behavioural activation system scales. European Journal of Personality, 15, 373-390. 
MacKinaw-Koons, B. \& Vasey, M. W. (2000). Considering sex differences in anxiety and its disorders across the lifespan: A construct validation approach. Applied and Preventative Psychology, 9, 191-209.

Mardaga, S. \& Hansenne, M. (2007). Relationships between Cloninger's biosocial model of personality and the behavioral inhibition/approach systems (BIS/BAS). Personality and Individual Differences, 42, 715-722.

Merckelbach, H., de Ruiter, C., \& Olff, M. (1989). Handedness and anxiety in normal and clinical populations. Cortex, 25, 599-606.

Perkins, A. M., Kemp, S. E. \& Corr, P. J. (2007). Fear and anxiety as seperable emotions: an investigation of the revised reinforcement sensitivity theory of personality. Emotion, 7(2), $252-261$.

Peters, M. (2008). Personal communication.

Peters, M. (1998). Description and validation of a flexible and broadly usable handedness questionnaire. Laterality, 3, 77-96.

Quilty, L. C., \& Oakman, J. M. (2004). The assessment of behavioural activation the relationship between impulsivity and behavioural activation. Personality and Individual Differences, 37 (2), 429-442. 
Quilty, L. C., Oakman, J. M. \& Farvolden, P. (2007). Behavioural inhibition, behavioural activation, and the preference for familiarity. Personality and Individual Differences, 42 (2), 291-303.

Schmidt, L. A., Fox, N. A., Schulkin, J., \& Gold, P. W. (1999). Behavioral and psychophysiological correlates of self-presentation in temperamentally shy children. Developmental Psychobiology, 35, 119-135.

Scholton, M. R. M., van Honk, J., Aleman, A., \& Kahn, R. S. (2006). Behavioral inhibition system (BIS), Behavioral activation system (BAS) and schizophrenia: Relationship with psychopathology and physiology. Journal of Psychiatric Research, 40 (7), 638-645.

Smillie, L. D., Pickering, A. D. \& Jackson, C. J. (2006). The new Reinforcement Sensitivity Theory: Implications for personality measurement. Personality and Social Psychology Review, 10, 320 - 335.

Stuettgen, M. C., Hennig, J., Reuter, M., \& Netter, P. (2005). Novelty seeking but not BAS is associated with high dopamine as indicated by a neurotransmitter challenge test using mazindol as a challenge substance. Personality and Individual Differences, 38 (7). 1597-1608.

Suhr, J. A. \& Tsanadis, J. (2007). Affect and personality correlates of the lowa Gambling Task. Personality and Individual Differences, 43 (1), 27-36. 
Sutton, S. K. \& Davidson, R. J. (1997). Prefrontal brain asymmetry: a biological substrate of the behavioral approach and inhibition systems. Psychological Science, 8, 204-210.

Wright, L. (2005). Response style differences between left- and right-handed individuals. PhD Thesis. University of Abertay Dundee.

Wright, L., Hardie, S., \& Rodway, P. (2004). Pause before you respond: Handedness influences response style on the Tower of Hanoi Task. Laterality, 9 (2), 133147. 
Table 1: Mean handedness scores (with standard deviations)

\begin{tabular}{ccc}
\hline & Left & Right \\
\hline Female & $-29.1(13.6)$ & $34.7(9.8)$ \\
Male & $-24(16.1)$ & $36.6(9.7)$ \\
Total & $-26.5(15)$ & $35.6(9.7)$ \\
\hline
\end{tabular}


Table 2: Means and standard deviations for BAS sub-section scores and total BAS scores for gender and handedness

\begin{tabular}{lccccccccc}
\hline & \multicolumn{2}{c}{ Male } & \multicolumn{2}{c}{ Female } & \multicolumn{2}{c}{ Total } & \multicolumn{3}{c}{ 2-way ANOVA } \\
& Left & Right & Left & Right & Left & Right & Hand & Gender & H x G \\
\hline BAS & 10.2 & 10.1 & 9.9 & 11.2 & 10.0 & 10.7 & 2.15 & $\mathrm{~F}<1$ & 2.57 \\
Drive & $(2.2)$ & $(2.1)$ & $(2.1)$ & $(2.3)$ & $(2.1)$ & $(2.3)$ & & & \\
BAS Fun & 11.8 & 11.9 & 10.6 & 11.4 & 11.2 & 11.7 & 1.65 & $4.70^{*}$ & 1.02 \\
Seeking & $(1.7)$ & $(2.1)$ & $(1.4)$ & $(2.2)$ & $(1.7)$ & $(2.2)$ & & & \\
BAS & 16.2 & 16.2 & 16.5 & 16.5 & 16.4 & 16.3 & $\mathrm{~F}<1$ & $\mathrm{~F}<1$ & $\mathrm{~F}<1$ \\
Reward & $(1.9)$ & $(1.8)$ & $(1.7)$ & $(2.5)$ & $(1.8)$ & $(2.2)$ & & & \\
BAS & 38.1 & 38.2 & 37.0 & 39.1 & 37.5 & 38.7 & 1.49 & $\mathrm{~F}<1$ & 1.43 \\
Total & $(3.8)$ & $(4.3)$ & $(3.8)$ & $(5.6)$ & $(3.8)$ & $(5.0)$ & & & \\
\hline${ }^{*} \mathrm{p}<0.05$ & & & & & & & & &
\end{tabular}


Table 3: Means and standard deviations for BIS scores for gender and handedness

\begin{tabular}{lccccccccc}
\hline & \multicolumn{2}{c}{ Male } & \multicolumn{2}{c}{ Female } & \multicolumn{2}{c}{ Total } & \multicolumn{2}{c}{ 2-way ANOVA } \\
& Left & Right & Left & Right & Left & Right & Hand & Gender & H x G \\
\hline BIS & 21.5 & 19.4 & 24 & 22.1 & 22.8 & 20.8 & $8.94^{* *}$ & $15.18^{\star *}$ & $\mathrm{~F}<1$ \\
scores & $(4.7)$ & $(2.0)$ & $(3.4)$ & $(3.6)$ & $(4.2)$ & $(3.3)$ & & & \\
${ }^{* \star} \mathrm{p}<0.01$ & & & & & & & & &
\end{tabular}


Table 4: Means and standard deviations for revised BIS (R-BIS) and FFFS scores for gender and handedness

\begin{tabular}{|c|c|c|c|c|c|c|c|c|c|}
\hline & \multicolumn{2}{|c|}{ Male } & \multicolumn{2}{|c|}{ Female } & \multicolumn{2}{|c|}{ Total } & \multicolumn{3}{|c|}{ 2-way ANOVA } \\
\hline & Left & Right & Left & Right & Left & Right & Hand & Gender & $H \times G$ \\
\hline & 15.6 & 14.3 & 17.5 & 15.8 & 16.6 & 15.1 & $7.54^{* *}$ & $9.54^{* *}$ & $\mathrm{~F}<1$ \\
\hline \multirow[t]{2}{*}{ R-BIS } & (3.9) & (2.0) & (2.6) & (2.9) & (3.4) & (2.6) & & & \\
\hline & 5.8 & 5.3 & 6.5 & 6.2 & 6.2 & 5.8 & 2.69 & $9.58^{* *}$ & $\mathrm{~F}<1$ \\
\hline FFFS & (1.7) & $(1.0)$ & (1.4) & (1.5) & (1.6) & (1.3) & & & \\
\hline
\end{tabular}

